\title{
UNDERSTANDING TeV-BAND COSMIC-RAY ANISOTROPY
}

\author{
Martin Pohl ${ }^{1,2}$ and David EICHLER ${ }^{3}$ \\ ${ }^{1}$ DESY, D-15738 Zeuthen, Germany; pohlmadq@ gmail.com \\ 2 Institute of Physics and Astronomy, University of Potsdam, D-14476 Potsdam, Germany \\ ${ }^{3}$ Physics Department, Ben-Gurion University, Be’er-Sheva 84105, Israel; eichler@bgu.ac.il \\ Received 2012 August 17; accepted 2013 January 29; published 2013 February 28
}

\begin{abstract}
We investigate the temporal and spectral correlations between flux and anisotropy fluctuations of TeV-band cosmic rays in light of recent data taken with IceCube. We find that for a conventional distribution of cosmic-ray sources, the dipole anisotropy is higher than observed, even if source discreteness is taken into account. Moreover, even for a shallow distribution of galactic cosmic-ray sources and a reacceleration model, fluctuations arising from source discreteness provide a probability only of the order of $10 \%$ that the cosmic-ray anisotropy limits of the recent IceCube analysis are met. This probability estimate is nearly independent of the exact choice of source rate, but generous for a large halo size. The location of the intensity maximum far from the Galactic Center is naturally reproduced.
\end{abstract}

Key word: cosmic rays

Online-only material: color figures

\section{INTRODUCTION}

The anisotropy in the arrival directions of cosmic rays in the $\mathrm{TeV}$ band has received renewed attention in recent years. A variety of experiments (Amenomori et al. 2006; Guillian et al. 2007; Aglietta et al. 2009; Abdo et al. 2009; Zhang et al. 2010; Abbasi et al. 2010, 2012; Di Sciascio et al. 2012) have reported results on the amplitude of the first harmonic in the sidereal anisotropy, which is typically found to be slightly less than $0.1 \%$. All of the experiments see only part of the sky, and it is not easy to reconstruct an all-sky dipole anisotropy, largely because the energy dependence of the acceptance is different among the various detectors. In any case, the true dipole anisotropy can be slightly higher than the sidereal first harmonic, i.e., $\lesssim 0.1 \%$. In addition, significant small-scale anisotropy was observed that has not found an accepted explanation to date, although it is tempting to attribute it to the local structure of the turbulent magnetic field in the Galaxy (e.g., Giacinti \& Sigl 2012; Giacinti et al. 2012).

Here, we investigate whether or not the dipole anisotropy can be reproduced with models of galactic cosmic-ray propagation that are tuned to fit available data on secondary-to-primary ratios and the survival fraction of unstable isotopes. For that purpose, we use a Green-function method that solves a time-dependent diffusion equation, similar to Blasi \& Amato (2012b). Diffusion equations of this type are derived by averaging the original Fokker-Planck transport equation for the isotropic part of the cosmic-ray distribution function. By construction, anisotropy in this treatment arises only from the diffusive (and possibly drift or convective) flux and is always dipolar in nature. Modeling the small-scale anisotropy, on the other hand, would be described by a transport equation derived from the original Fokker-Planck equation for the anisotropic part of the cosmic-ray distribution and is not considered here.

Our time-dependent treatment permits us to account for a discrete nature of cosmic-ray source. A similar technique was used to model variations in the spectrum of cosmic-ray electrons (Pohl \& Esposito 1998; Pohl et al. 2003; Mertsch 2011) and cosmic-ray ions (Büsching et al. 2005; Ptuskin et al. 2006; Blasi $\&$ Amato 2012a, 2012b). We test various cosmic-ray source dis- tributions in galactocentric radius, using the appropriate transport parameters, and compute the expected temporal evolution of the anisotropy. This approach permits a realistic assessment of the likelihood to find a certain anisotropy for a specific choice of parameters. The occasionally discussed mean variance of the anisotropy is a considerably less useful quantity, because the distribution of anisotropy amplitudes is highly skewed.

We are particularly interested in the temporal and spectral correlations between flux and anisotropy fluctuations. Our approach is ideally suited to recover these correlations and investigate their effect when we compute flux and anisotropy over a finite spectral acceptance and compare with observational results.

\section{CALCULATION OF PARTICLE SPECTRA}

\subsection{Cosmic-ray Ions}

The differential density of $\mathrm{TeV}$-band cosmic rays obeys a continuity equation that can be written as

$$
\frac{\partial N}{\partial t}-\frac{1}{r^{2}} \frac{\partial}{\partial r}\left(r^{2} D \frac{\partial N}{\partial r}\right)=Q(E) \delta(t) \frac{\delta(r)}{4 \pi r^{2}} .
$$

Here, $D=c \lambda_{\mathrm{mfp}} / 3$ is the spatial diffusion coefficient, $r$ is the distance from the source, and $Q(t, E)$ is the differential production rate at the source. Note that we neglect stochastic reacceleration, advection, and energy losses. The first and the last of that list should only be important at $\mathrm{GeV}$ energies and below, at least for cosmic-ray protons. The case of advection is less clear, but probably also less important than it may be in the $\mathrm{GeV}$ band on account of the energy dependence of diffusive propagation.

Using standard methods (Kardashev 1962; Ginzburg \& Syrovatskii 1964), we derive a general solution to the transport equation, assuming impulsive particle injection at time $t=0$,

$$
N(r, t, E)=\frac{\Theta(t)}{(4 \pi D t)^{3 / 2}} Q(E) \exp \left(-\frac{r^{2}}{4 D t}\right) .
$$

If more than one source contributes to the local cosmic-ray flux at any time, then their individual contributions must be calculated using Equation (2) and then summed. 
We now modify the solution to account for a finite size and lifetime of sources and for escape from the Galaxy. It is known that assuming a point source in space and time can lead to singularities in the cosmic-ray density (e.g., Mertsch 2011). Real cosmic-ray sources, whatever their nature, have a finite spatial extent and confine the accelerated particles for some time because the acceleration is not instantaneous. The most simple method to represent extended sources is to assume that they have the shape of a thin, spherical shell of radius $R$. The correspondence of a young supernova remnant (SNR) to the shell is obvious, but the applicability of the ansatz is not limited to SNR. In any case, averaging over the shell yields

$$
\begin{aligned}
N(r, t, E)= & \frac{\Theta(t)}{(4 \pi D t)^{3 / 2}} Q(E)\left[\frac{D t}{r R}\right] \\
& \times \sum_{ \pm}( \pm 1) \exp \left(-\frac{(r \mp R)^{2}}{4 D t}\right) .
\end{aligned}
$$

Note that this solution still has a singularity for $t \rightarrow 0$ at $r=R$, which will disappear when we average over a finite lifetime, because asymptotically $N \propto t^{-0.5}$ for $t \rightarrow 0$.

We do need to account for source size in computing the anisotropy, which is given by the diffusive flux,

$$
\delta=-\frac{\lambda_{\mathrm{mfp}}}{N} \frac{\partial N}{\partial r}=\frac{\lambda_{\mathrm{mfp}}}{r}+\frac{3 r}{2 c t}-\frac{3 R}{2 c t} \operatorname{coth}\left(\frac{r R}{2 D t}\right) .
$$

For small arguments of the coth term, the first and last terms on the right-hand side cancel each other.

For the integration over the time of source activity, we choose the most simple form of a temporal profile, namely a constant source rate over an age interval $\left[T_{1} ; T_{2}\right]$, with the constraint that both $T_{1}$ and $T_{2}$ be positive. The time integral can be easily performed analytically, but leads to very small differences of exponentials and error functions that are more difficult to numerically implement than a simple numerical integration. A fact that we shall employ below is that either escape is irrelevant or the effects of the finite extent of the lifetime of the sources are negligible, provided that the source lifetime is much smaller than the escape time.

Diffusive escape from the Galaxy can be modeled using Dirichlet boundary conditions at some distance from the midplane of the Galaxy, $N(z= \pm H)=0$. Obviously, the boundary conditions break the spherical symmetry of the problem. Whereas for very rare sources analytical approximations may ease the treatment (Pohl \& Eichler 2011), in the TeV band we expect cosmic-ray sources to appear with a rate $\sim 1 /(100 \mathrm{yr})$, implying a relatively small distance to the nearest source. Consequently, we attempt a full solution by using the method of mirror sources. Since we have two boundaries, an infinite series of mirror sources with alternating sign is needed (Blasi \& Amato 2012a).

For the mirror sources, only the Gaussian factor in Equation (3) is relevant. It is accurate to second order in the ratio of source lifetime and escape time to use an average age, $\tau=\left(T_{2}+T_{1}\right) / 2$. Mirror sources modify the $z$-dependent Gaussian factor in Equation (3) as

$$
\begin{aligned}
& \exp \left[-\frac{\left(z-z_{s}\right)^{2}}{4 D \tau}\right] \rightarrow G=\sum_{n=-\infty}^{\infty}(-1)^{n} \\
& \quad \times \exp \left[-\frac{\left[z-2 n H-(-1)^{n} z_{s}\right]^{2}}{4 D \tau}\right]
\end{aligned}
$$

which, as is described in the Appendix, can be approximated with good accuracy as

$$
\begin{aligned}
G & \simeq \exp \left[-\frac{\left(z-z_{s}\right)^{2}}{4 D \tau}\right](1+1.5 x)^{1.25} \\
& \times \exp \left[-(1.5 x)^{0.97}\right] \quad x=\frac{2 D \tau}{H^{2}} .
\end{aligned}
$$

\subsection{Cosmic-ray Electrons}

It is well known that the flux and spectrum of cosmic-ray electrons from discrete sources are particularly sensitive to fluctuations (Pohl \& Esposito 1998; Pohl et al. 2003), but the consequences for the electron anisotropy are rarely discussed in the literature. In fact, the anisotropy in the intensity of cosmicray electrons at energies between $60 \mathrm{GeV}$ and $480 \mathrm{GeV}$ has only recently been measured (Ackermann et al. 2010). In the case of electrons, we need to account for their energy losses, but may neglect escape, at least above $\sim 60 \mathrm{GeV}$. Hence, we may describe the propagation of electrons at energies higher than a few $\mathrm{GeV}$ by a simplified, time-dependent transport equation:

$$
\frac{\partial N_{e}}{\partial t}-\frac{\partial}{\partial E}\left(b E^{2} N_{e}\right)-D E^{a} \nabla^{2} N_{e}=Q_{e},
$$

where we consider continuous energy losses by synchrotron radiation and inverse Compton scattering, a diffusion coefficient $D_{0} E^{a}$ dependent on energy, and a source term, $Q_{e}$. Green's function for this problem can be found in the literature (Ginzburg \& Syrovatskii 1964):

$$
G_{e}=\frac{\delta\left(t-t^{\prime}+\frac{E-E^{\prime}}{b E E^{\prime}}\right)}{b E^{2}(4 \pi \lambda)^{3 / 2}} \exp \left(-\frac{\left(r-r^{\prime}\right)^{2}}{4 \lambda}\right)
$$

with

$$
\lambda=\frac{D_{0}\left(E^{a-1}-E^{\prime a-1}\right)}{b(1-a)} .
$$

As in the case of cosmic-ray ions, we assume that the individual sources inject a spectrum $\propto E^{-s}$ at constant rate for a defined period of time, and so the average over the age from $T_{1}$ to $T_{2}$ is

$$
N_{e}=q_{e} E^{-s} \int_{0}^{\frac{1}{b E}} d t^{\prime} \frac{\Theta\left(t^{\prime}-T_{1}\right) \Theta\left(T_{2}-t^{\prime}\right)}{(4 \pi \Lambda)^{3 / 2}\left(1-b E t^{\prime}\right)^{2-s}} \exp \left(-\frac{r^{2}}{4 \Lambda}\right) \text {, }
$$

where

$$
\Lambda=\frac{D_{0} E^{a-1}}{b(1-a)}\left(1-\left(1-b E t^{\prime}\right)^{1-a}\right)
$$

and $r$ is the distance between source and observer.

As in the case of ions, we can easily average over a shell of radius $R$ and obtain

$$
\begin{aligned}
N_{e}= & q_{e} E^{-s} \int_{0}^{\frac{1}{b E}} d t^{\prime} \frac{\Theta\left(t^{\prime}-T_{1}\right) \Theta\left(T_{2}-t^{\prime}\right)}{(4 \pi \Lambda)^{3 / 2}\left(1-b E t^{\prime}\right)^{2-s}} \\
& \times\left[\frac{\Lambda}{r R}\right] \sum_{ \pm}( \pm 1) \exp \left(-\frac{(r \pm R)^{2}}{4 \Lambda}\right) .
\end{aligned}
$$

A general analytical solution is very difficult to obtain. However, an explicit integration is unnecessary if $\left(T_{2}-T_{1}\right) \ll T_{1}$, provided the energy-dependent upper limit of integration is observed. If 


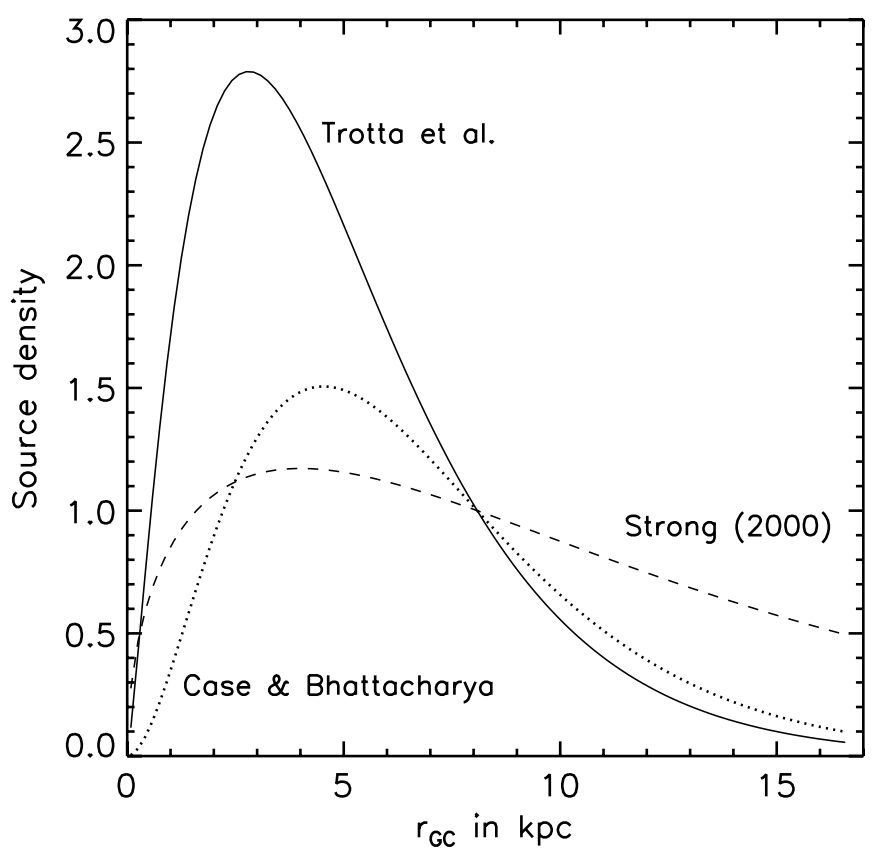

Figure 1. Distribution of the cosmic-ray source density in galactocentric radius, according to the three estimates used in the literature. All curves are normalized to unity at the solar circle.

$s>2$, as is the case for cosmic-ray electrons, then the integrand approaches zero at $t \rightarrow(b E)^{-1}$. Then, it is accurate to second order in $\left(T_{2}-T_{1}\right) / T_{1}$ to set

$$
\begin{aligned}
N_{\mathrm{av}, e}= & q_{e} E^{-s} \frac{1}{(4 \pi \Lambda)^{3 / 2}(1-b E \tau)^{2-s}} \\
& \times\left[\frac{\Lambda(\tau)}{r R}\right] \sum_{ \pm}( \pm 1) \exp \left(-\frac{(r \pm R)^{2}}{4 \Lambda(\tau)}\right),
\end{aligned}
$$

where $\tau=\left(T_{2}+T_{1}\right) / 2$. The energy-loss time of electrons at $500 \mathrm{GeV}$ in a $10 \mu \mathrm{G}$ magnetic field is about $3 \times 10^{5} \mathrm{yr}$, much longer than the source lifetime $T_{2}-T_{1}$ that we consider here. If $b E t$ is a small parameter, then we can expand the integrand in Equation (12). By noting that for $b E t \ll 1$

$$
\Lambda \simeq D_{0} E^{a} t
$$

we find that Equation (12) assumes the same form as the time integral over Equation (3), the corresponding expression for cosmic-ray ions.

\section{INTERMITTENCY AND ANISOTROPY}

Unless the number of cosmic-ray sources is very small, and hence intermittency effects dominate, the source distribution is a critical factor in anisotropy studies. Often, a function of the type

$$
Q\left(r_{\mathrm{GC}}\right)=\left(\frac{r_{\mathrm{GC}}}{R_{\odot}}\right)^{a} \exp \left(-b \frac{r_{\mathrm{GC}}-R_{\odot}}{R_{\odot}}\right)
$$

is used to represent the density distribution in galactocentric radius, $r_{\mathrm{GC}}$. The parameters $a$ and $b$ are derived by fitting observed distributions of pulsars (Lorimer 2004), SNRs (Case \& Bhattacharya 1998), or other perceived tracers of cosmic-ray acceleration.

It was noted earlier that the observed intensity of diffuse Galactic gamma rays appears to require a substantially flatter
Table 1

Standard Parameters, Mostly Taken from the Study of Trotta et al. (2011)

\begin{tabular}{lcc}
\hline \hline Parameters & Symbol & Value \\
\hline Injection index & $s$ & 2.4 \\
Energy dependence of diffusion & $\delta$ & 0.3 \\
Source distribution & $a$ & 1.25 \\
Source distribution & $b$ & 3.56 \\
Source rate & $P_{Q}$ & $10^{-2} \mathrm{yr}^{-1}$ \\
Source lifetime & & $2 \times 10^{3} \mathrm{yr}$ \\
Source radius & $R$ & $10 \mathrm{pc}$ \\
\hline
\end{tabular}

source distribution (Strong et al. 2000), although a metallicity gradient, and hence a gradient in the $X_{\mathrm{CO}}$ conversion factor of CO line intensity, could explain the gamma-ray data as well (Strong et al. 2004). The comprehensive analysis of Fermi-LAT data by Ackermann et al. (2012) also suggests a gradient in $X_{\mathrm{CO}}$, albeit a weaker trend as in Strong et al. (2004). A similar conclusion was reached by Ackermann et al. (2011).

Here, we select from the literature three models of the cosmicray source distribution in the Galaxy. Our sample comprises the relatively flat distribution used by Strong et al. (2000), the SNR distribution derived by Case \& Bhattacharya (1998), which is slightly flatter than that of Green (2012), and the pulsar distribution as presented by Trotta et al. (2011), which is close to that of Lorimer (2004). Figure 1 permits a visual comparison of the three models.

According to Trotta et al. (2011), which we choose for the definition of standard parameters, we have well-constrained propagation parameters, such as the injection index $s=2.4 \pm$ 0.1 , and for the diffusion coefficient

$$
D=D_{0}\left(\frac{E}{4 \mathrm{GeV}}\right)^{\delta}
$$

with $\delta=0.3 \pm 0.05$ in reacceleration models, whereas a degeneracy arises with the halo size. In units of kpc, $3 \leqslant H \leqslant 9$, and

$$
D_{0} \simeq(1.2+1.3 H) \times 10^{28} \mathrm{~cm}^{2} \mathrm{~s}^{-1}
$$

within about $20 \%$ error margin. We try four values of $H$ and derive a $95 \%$ confidence minimum of anisotropy in the 10-100 TeV band. Standard parameters are summarized in Table 1. Note that these parameters were determined using the sharply peaked source distribution labeled Trotta et al. in Figure 1. The anisotropy and its variation are shown as a function of the particle energy in Figure 2, based on 100 randomly selected samples of cosmic-ray sources. As expected, the range of probable anisotropy values slowly increases with energy. Something to note from Figure 2 is the skewness in the distribution of anisotropy levels, which renders the use of a mean fluctuation amplitude in the anisotropy less than useful. In fact, the distribution of anisotropy levels is approximately symmetric in $\log (\delta)$. Statistical fluctuations cause jitter in the limits of the $90 \%$ containment band, but we can see that the width of the fluctuation range does not change with energy by more than a factor two over four decades in energy.

Our full results, based on 10,000 Monte Carlo runs for each parameter combination, thus minimizing the statistical noise, are detailed in Table 2. We note that the anisotropy increases with halo size and decreases if the radial distribution of cosmic-ray sources is assumed to be shallow (as with Strong et al. 2000).

It is obvious that when using the viable propagation parameters of Trotta et al. (2011), the limits on anisotropy may be met 


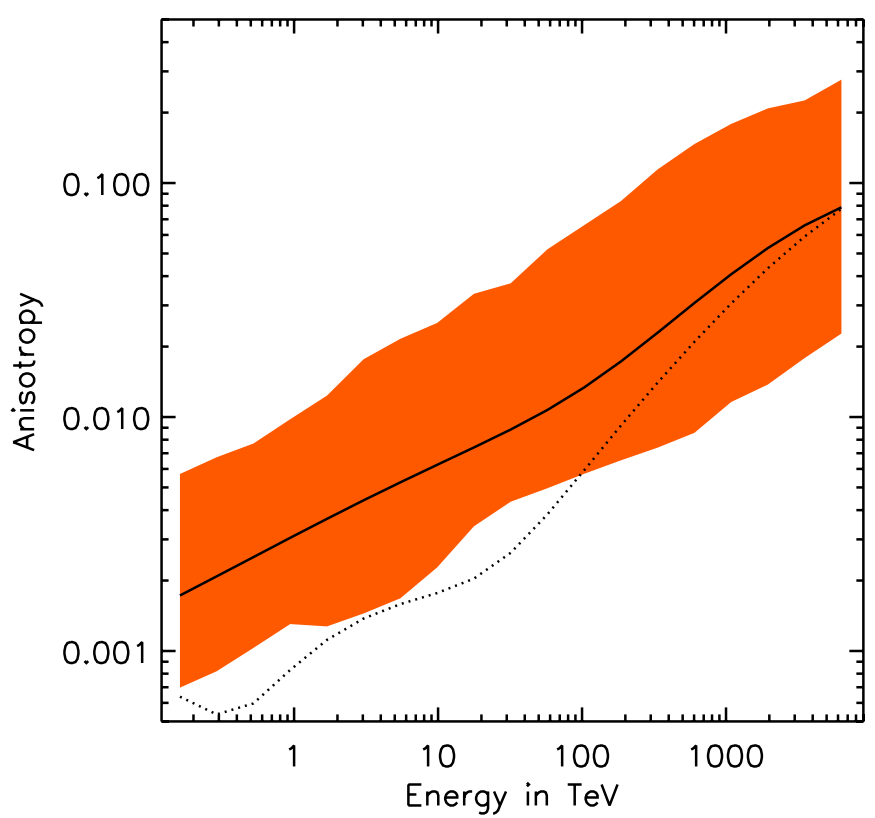

Figure 2. Anisotropy for standard parameters (Trotta et al.) and $H=5 \mathrm{kpc}$. The red band indicates the central $90 \%$ of the fluctuation range. The solid line marks the median of the distribution and the dotted line describes the anisotropy amplitude in a randomly selected run.

(A color version of this figure is available in the online journal.)

Table 2

Proton Anisotropy at $20 \mathrm{TeV}$ Total Energy for Various Parameters and Four Choices of Halo Size, $H$ in kpc

\begin{tabular}{lcllll}
\hline \hline Parameters & Confidence & $H=3$ & $H=5$ & $H=7$ & $H=9$ \\
\hline Standard & 95\% lower limit & $0.27 \%$ & $0.37 \%$ & $0.45 \%$ & $0.53 \%$ \\
Standard & Median & $0.64 \%$ & $0.76 \%$ & $0.87 \%$ & $0.97 \%$ \\
$\delta=0.25$ & 95\% lower limit & $0.18 \%$ & $0.24 \%$ & $0.30 \%$ & $0.34 \%$ \\
$\delta=0.25$ & Median & $0.42 \%$ & $0.49 \%$ & $0.57 \%$ & $0.64 \%$ \\
Case \& Bhattacharya & 95\% lower limit & $0.16 \%$ & $0.22 \%$ & $0.27 \%$ & $0.32 \%$ \\
Case \& Bhattacharya & Median & $0.51 \%$ & $0.59 \%$ & $0.67 \%$ & $0.75 \%$ \\
Strong (2000) & 95\% lower limit & $0.085 \%$ & $0.094 \%$ & $0.11 \%$ & $0.13 \%$ \\
Strong (2000) & Median & $0.38 \%$ & $0.38 \%$ & $0.37 \%$ & $0.44 \%$ \\
\hline
\end{tabular}

only if either the TeV-band cosmic rays are relatively heavy or the source distribution is very flat. A shallow source distribution is certainly possible, but it was not the one used to find the propagation parameters best suited to reproduce $\mathrm{B} / \mathrm{C}$ or ${ }^{10} \mathrm{Be}$ data.

\subsection{Shallow Source Distribution}

To verify the reliability of our findings, we test them against the results of Putze et al. (2010), who used a different propagation model and a constant source density. We do not use their reacceleration/convection model, because in two-dimensional geometry with radial boundary the best-fit parameters indicate that advection has little importance above a TeV.

For the reacceleration model, the scaling of the diffusion coefficient with halo size (in $\mathrm{kpc}$ ) is

$$
D_{0} \simeq H 1.04 \times 10^{28} \mathrm{~cm}^{2} \mathrm{~s}^{-1}
$$

with $1.5 \leqslant H \leqslant 6$. Numerically, the scale factor of the diffusion coefficient is similar to that of Trotta et al. (2011), but at $20 \mathrm{TeV}$ the diffusion coefficient is actually a factor of 2.6 smaller on account of the weaker energy dependence of diffusion. Other parameters are summarized in Table 3 . We use the relatively flat

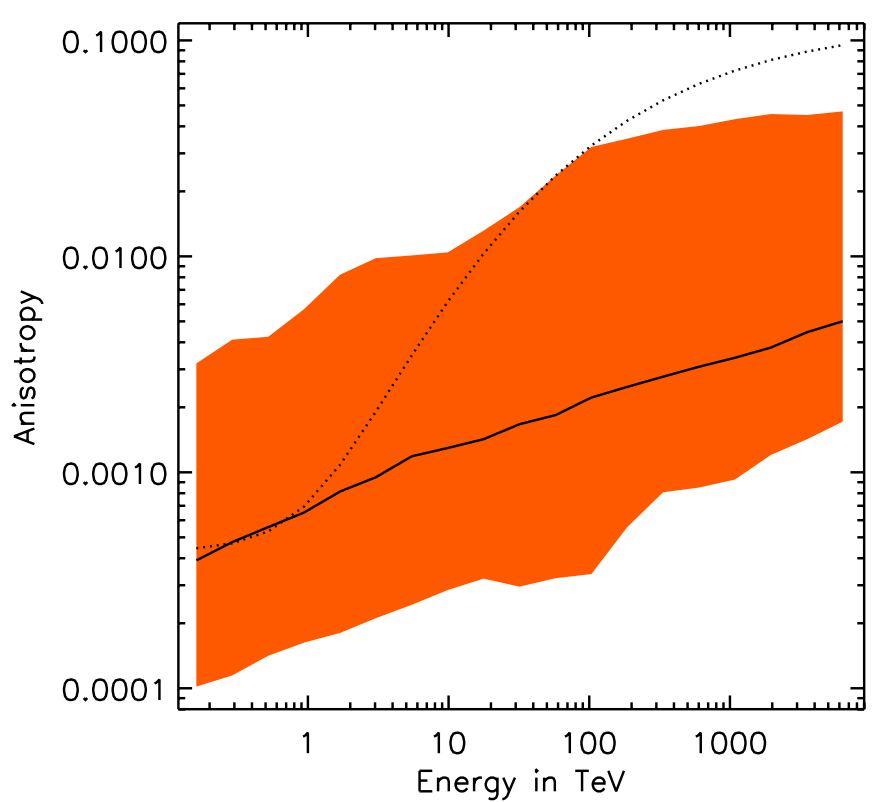

Figure 3. Anisotropy for a shallow source distribution with parameters as listed in Table 3 and halo size $H=4.5 \mathrm{kpc}$. As in Figure 2, the red band indicates the central $90 \%$ of the fluctuation range, the solid line marks the median of the distribution, and the dotted line describes the anisotropy amplitude in a randomly selected run.

(A color version of this figure is available in the online journal.)

Table 3

Parameters for a Shallow Source Distribution, Mostly Taken from Putze et al.

\begin{tabular}{lcc}
\hline \hline Parameters & Symbol & Value \\
\hline Injection index & $s$ & 2.415 \\
Energy dependence of diffusion & $\delta$ & 0.235 \\
Source rate & $P_{Q}$ & $10^{-2} \mathrm{yr}^{-1}$ \\
Source lifetime & & $2 \times 10^{3} \mathrm{yr}$ \\
Source radius & $R$ & $10 \mathrm{pc}$ \\
\hline
\end{tabular}

Table 4

Proton Anisotropy at $20 \mathrm{TeV}$ Total Energy for a Shallow Source Distribution

\begin{tabular}{lcllll}
\hline \hline Parameters & Confidence & $H=1.5$ & $H=3$ & $H=4.5$ & $H=6$ \\
\hline Standard & $95 \%$ lower limit & $0.027 \%$ & $0.030 \%$ & $0.035 \%$ & $0.042 \%$ \\
Standard & Median & $0.13 \%$ & $0.10 \%$ & $0.13 \%$ & $0.16 \%$ \\
Source rate & $95 \%$ lower limit & $0.030 \%$ & $0.031 \%$ & $0.036 \%$ & $0.041 \%$ \\
$P_{Q}=10^{-3} \mathrm{yr}^{-1}$ & Median & $0.14 \%$ & $0.14 \%$ & $0.15 \%$ & $0.16 \%$ \\
Source rate & $95 \%$ lower limit & $0.037 \%$ & $0.034 \%$ & $0.039 \%$ & $0.041 \%$ \\
$P_{Q}=10^{-4} \mathrm{yr}^{-1}$ & Median & $0.17 \%$ & $0.15 \%$ & $0.16 \%$ & $0.17 \%$ \\
\hline
\end{tabular}

source distribution of Strong et al. (2000) out to $r_{\mathrm{GC}}=20 \mathrm{kpc}$, and henceforth refer to it as the shallow source distribution.

In Figure 3, we show the distribution of anisotropy levels as a function of energy, similar to Figure 2 and likewise based on 100 source samples. The full results based on $10^{4}$ Monte Carlo runs for each parameter set are summarized in Table 4. Something to note from the table is the strong reduction in anisotropy resulting from the moderate (factor 2.6) decrease in the diffusion coefficient (see Table 2, in particular the runs for Strong et al. (2000)). There is, however, only a weak, if any, trend with either halo size or source rate. 


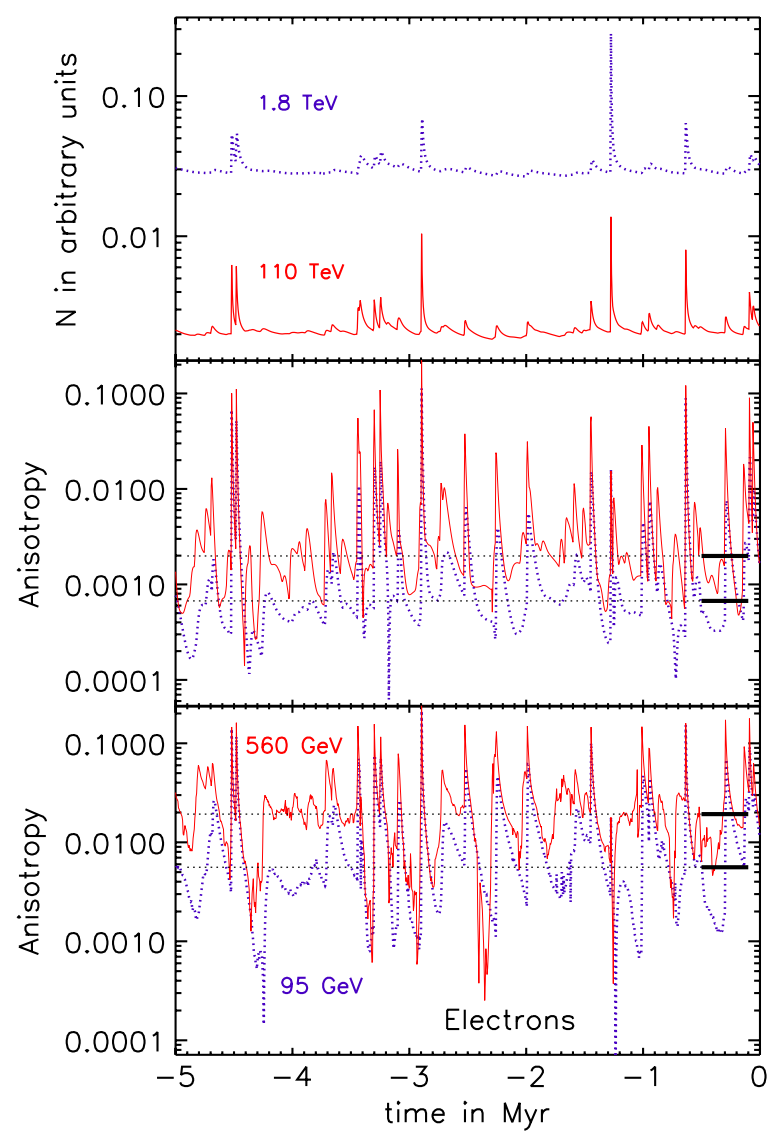

Figure 4. Light curves of proton flux at two energies (top panel), proton anisotropy (middle panel), and electron anisotropy at two lower energies (bottom panel). The black bars and dotted horizontal lines indicate the median of the anisotropies.

(A color version of this figure is available in the online journal.)

\section{LIGHT CURVES}

\subsection{General Behavior}

Having established that a rather flat source distribution is necessary to explain the low anisotropy amplitude measured at multi-TeV energies, we now discuss light curves. We want to infer the temporal and spectral correlations between flux and anisotropy fluctuations, which must be taken into account when computing the flux and anisotropy over a finite spectral acceptance and comparing with observational results.

A number of insights can be gained by first discussing light curves at specific energies. Figure 4 shows the temporal variations at $5 \mathrm{kyr}$ resolution of the proton flux, proton anisotropy, and electron anisotropy at two energies for the standard parameters with halo size $H=3 \mathrm{kpc}$ and a shallow source distribution (see Table 3). Our findings can be summarized as follows.

1. The proton flux displays small-amplitude fluctuations with rare, short-lived spikes, which is a result already discussed by Büsching et al. (2005).

2. Spikes in flux are generally accompanied by spikes in anisotropy at all energies and reflect the appearance with nearby, young cosmic-ray sources. The events may change the diffusion coefficient on account of streaming instabilities, in which case this calculation would overestimate the anisotropy during spikes.

3. Deep, short-lived dips can be observed in the anisotropy, which have no counterpart in the flux and do not appear at

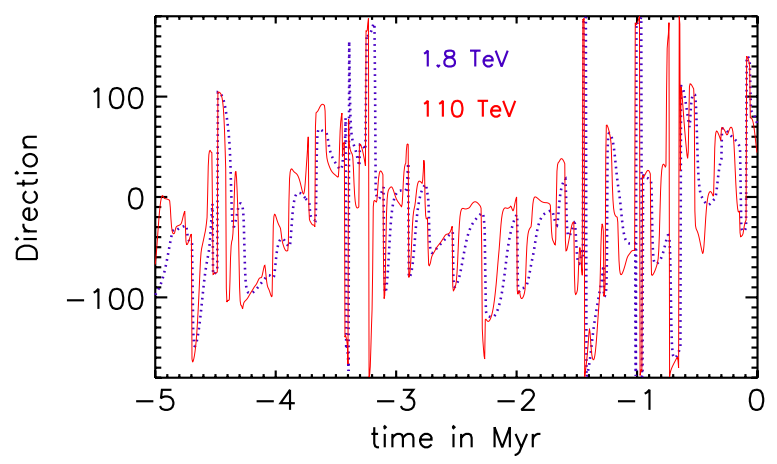

Figure 5. Distribution in Galactic longitude of the direction of maximum proton intensity.

(A color version of this figure is available in the online journal.)

all energies. These dips arise from a random balancing of the contributions of the dominant cosmic-ray sources and are therefore a hallmark of discrete sources.

4. There is little correlation between the small-amplitude fluctuations in the anisotropy at different energies, which implies that care must be exercised when comparing with airshower data, which carry contributions from cosmic rays over a wide range of energies.

5. The predicted electron anisotropy is lower than the upper limits published by Ackermann et al. (2010), but higher by a factor $\sim 5$ than the GALPROP predictions shown in their Figure 9. GALPROP does not properly account for discrete sources and therefore would underpredict the anisotropy.

The direction of maximum intensity is almost always in the Galactic plane, because the distribution of cosmic-ray sources with the vertical coordinate, $z$, is very narrow. In Figure 5, we show how the anisotropy direction varies with time, using the same simulation as for Figure 4 for ease of comparison. Something to note from the figure is the correspondence of the deep dip in anisotropy at $-3.2 \mathrm{Myr}$ with a $360^{\circ}$ swing of the anisotropy direction. Generally, the variations in the anisotropy direction are large and not achromatic, although the difference in directions at $1.8 \mathrm{TeV}$ and $110 \mathrm{TeV}$ rarely exceeds $30^{\circ}$.

Ground-based observatories do not see the entire sky, and therefore resort to measuring the first harmonic in right ascension while integrating over the declination band that is accessible to them. The projection of a first harmonic in galactic longitude depends on the direction of maximum intensity and on the declination band in question.

\subsection{Comparison with IceCube Results}

The IceCube collaboration has presented cosmic-ray anisotropy measurements in two broad energy bands (Abbasi et al. 2012) based on a total of about 30 billion cosmic-rayinduced muon events. Airshowers caused by heavy primaries approximately behave like the superposition of airshowers produced by the individual nucleons. Therefore, the detection with IceCube of a muon with a certain energy requires a heavy primary of much higher energy than it would if the primary were a proton. In terms of propagation, heavy cosmic rays with charge number $Z$ and energy $E$ will behave like protons of energy $E / Z$. Thus, light and heavy cosmic-ray nuclei that have similar propagation properties, and hence similar anisotropy, will also account for muons of similar energy in IceCube. We have seen in Figure 3 that a factor of two in energy yields a 20\% change in anisotropy on average. We have also seen that the spectrum of 


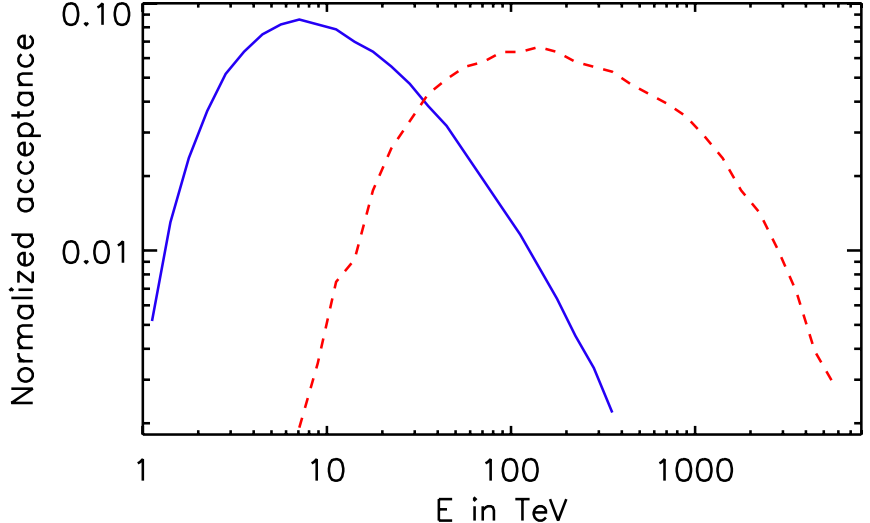

Figure 6. Distribution in energy of the primary particle for the high-energy (red line) and low-energy (blue line) event selections, derived under the assumption that all primary cosmic rays are protons.

(A color version of this figure is available in the online journal.)

cosmic rays shows little variation, except possibly during intense spikes in flux. It is therefore a good approximation to only consider cosmic-ray protons. Nevertheless, we shall indicate in the subsequent text the changes in the main results that would arise had we assumed that all of the primary particles were helium nuclei.

The IceCube collaboration was kind enough to provide us with the distribution in energy of the primary particle for their two muon event selections, derived under the assumption that all primary cosmic rays are protons. These distributions are also shown in Figure 6. Obviously, the median primary particle energies are lower than for the mixed composition assumed in Abbasi et al. (2012), but these are the distributions we need to combine with our proton-propagation calculation described in the preceding sections. If all of the primary cosmic rays were helium nuclei, then the corresponding distributions would be shifted to higher energies. Figure 4 in Abbasi et al. (2012) indicates that for their low-energy sample, the up-shift in energy is approximately a factor of four in total energy, i.e., helium primaries have about twice the rigidity of proton primaries.

IceCube reports the sidereal first harmonic in the cosmicray intensity averaged over the declination range $-25^{\circ}<\theta<$ $-72^{\circ}$. Obviously, the dependence of acceptance on the primary energy is not constant over this declination range, but we will ignore that and project the cosmic-ray dipole anisotropy onto the sidereal band at the characteristic declination $\theta_{c}=-45^{\circ}$, which is approximately the median declination of events accepted in the IceCube analysis. Note that we compute the direction of the cosmic-ray dipole anisotropy (see Figure 5), which displays considerable variability, as does its projection onto the sidereal band.

A dipole anisotropy of amplitude $\delta$ pointing at declination $\theta_{d}$ provides a sidereal first harmonic at declination $\theta_{c}$ of amplitude

$$
\delta_{\text {proj }}\left(\theta_{c}\right)=\frac{\delta \cos \theta_{d} \cos \theta_{c}}{1+\delta \sin \theta_{d} \sin \theta_{c}} .
$$

The denominator can essentially always be set to unity. We simulate the cosmic-ray intensity for $50 \mathrm{Myr}$ with $5 \mathrm{kyr}$ resolution to obtain a statistically meaningful data set. In Figure 7, we show the expected anisotropy in the IceCube data over $20 \mathrm{Myr}$ only for a source rate $P_{Q}=3 \times 10^{-3} \mathrm{yr}^{-1}$. As the anisotropy direction varies with energy, the broad spectral acceptance of the IceCube data selection leads to a reduction in

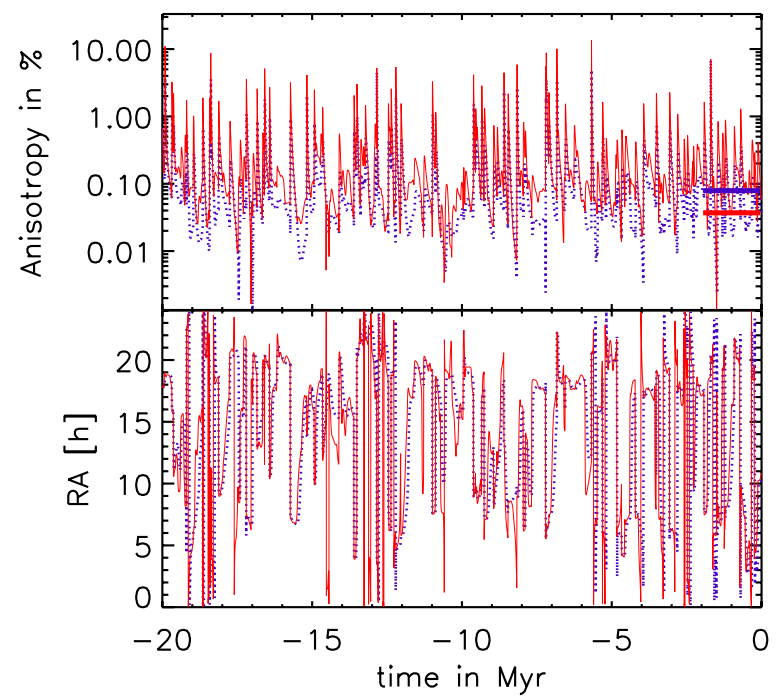

Figure 7. Top panel: expected sidereal anisotropy in IceCube data at declination $\theta_{c}=-45^{\circ}$ for the high-energy selection (in red) and low-energy band (in blue). The bars on the right indicate measured limits. The source rate is assumed to be one per thousand years. Bottom panel: location in right ascension of the maximum intensity in IceCube data for the high-energy selection (in red) and low-energy band (in blue).

(A color version of this figure is available in the online journal.)

the expected anisotropy level, i.e., models are brought closer to the data, which we explicitly account for in our calculations.

The IceCube collaboration reports the following sidereal anisotropies:

$$
\begin{aligned}
\delta_{\text {obs }}(\text { low energy }) & =\left(7.9 \pm 0.1_{\text {stat }} \pm 0.3_{\text {sys }}\right) \times 10^{-4} \\
\delta_{\text {obs }}(\text { high energy }) & =\left(3.7 \pm 0.7_{\text {stat }} \pm 0.7_{\text {sys }}\right) \times 10^{-4}
\end{aligned}
$$

The expected anisotropy is simultaneously below both values for $8.2 \%$ of the simulated time period of $50 \mathrm{Myr}$. If we assume helium primaries instead of protons and retain their spectrum as in Hörandel (2003), then both anisotropy values are met for only $6.6 \%$ of the simulated time. If, in addition, we assume that the spectrum of helium nuclei is harder than that of proton nuclei by $\Delta s=0.08$, as is suggested by data taken with CREAM (Yoon et al. 2011), then the expected anisotropy is below that measured at only $6.1 \%$ of the time. We can conclude that cosmicray helium is less likely to yield the low observed anisotropy signal in the IceCube data than are cosmic-ray protons.

Even if we account for the uncertainties and set the anisotropies to $8.2 \times 10^{-4}$ and $4.8 \times 10^{-4}$, respectively, implying that individually there is only a $16 \%$ probability that the true anisotropy is higher, they are only met at $12.9 \%$ of the time for proton primaries, and less than that for helium primaries.

Also shown in Figure 7 is the location of peak intensity in the sidereal band studied by IceCube. Something to note from the figure is that, in particular, during anisotropy lows, the peak intensity is found far from the Galactic-Center direction at R.A. $\simeq 17^{\text {h }} 8$ and not necessarily coincident for the two energy bands. Both results reproduce the IceCube observations.

To verify that this result does not significantly depend on the assumed source rate and halo size, we have varied the rate between $P_{Q}=3 \times 10^{-4} \mathrm{yr}^{-1}$ and $P_{Q}=3 \times 10^{-2} \mathrm{yr}^{-1}$, and the halo size between $H=3 \mathrm{kpc}$ and $H=7 \mathrm{kpc}$. In no case was the probability to simultaneously meet both anisotropy values higher than $11 \%$. This corresponds to approximately 1000 out of the 10,000 point in the light curve. We estimate 
Table 5

Summary of Anisotropy Results

\begin{tabular}{lccccc}
\hline \hline $\begin{array}{l}\text { Rate } \\
\left(10^{3} \mathrm{Myr}^{-1}\right)\end{array}$ & $\begin{array}{c}\text { Halo } \\
(\mathrm{kpc})\end{array}$ & $\begin{array}{c}\text { Prob. } \\
(\%)\end{array}$ & $\begin{array}{c}\text { Med. LE } \\
\left(10^{-4}\right)\end{array}$ & $\begin{array}{c}\text { Med. HE } \\
\left(10^{-4}\right)\end{array}$ & Line \\
\hline 0.3 & 3 & 8.2 & 7.1 & 13.5 & Black \\
0.3 & 7 & 3.3 & 8.3 & 15.7 & Blue dotted \\
3 & 7 & 4.2 & 7.7 & 15.2 & Red dotted \\
3 & 3 & 8.2 & 6.8 & 12.8 & Red \\
1 & 3 & 10.4 & 6.8 & 13.2 & Blue \\
10 & 3 & 9.1 & 6.2 & 12.0 & Green \\
\hline
\end{tabular}

Notes. The first two columns give the assumed source rate and halo size. The next three columns give the probability that the anisotropy is below that measured with IceCube, and the anisotropy median at low energy and high energy. The last column identifies the corresponding lines in Figure 8.

that the statistical uncertainty does not exceed 1 percentage point. Table 5 summarizes the results, and Figure 8 displays the integral probability to measure an anisotropy lower than a certain threshold, $A$. To be noted from the figure is that varying the source rate does not change the probability distribution in a significant manner, but choosing a large halo size appears to reduce the probability of a low anisotropy.

We can also test the effect of an energy-scale error in the IceCube data. If we suppose that the cosmic-ray primaries responsible for the muons seen with IceCube have an energy $25 \%$ lower than estimated, shown in Figure 6, then the probabilities to reproduce the reported sidereal anisotropy increases between 0.5 and 1 percentage point. The highest probability for any tested parameter combination is $11.2 \%$.

As a last test, we repeated one simulation $(H=3 \mathrm{kpc}$ and $P_{Q}=3 \times 10^{-3} \mathrm{yr}^{-1}$ ) for a perfectly flat source distribution out to $r_{\mathrm{GC}}=20 \mathrm{kpc}$. The probability of meeting the IceCube upper limits was $11 \%$ (11.9\% with a shift in energy scale), only marginally larger than for the shallow source distribution of Strong et al. (2000).

\section{DISCUSSION}

This is not the first calculation of cosmic-ray anisotropy in the $\mathrm{TeV}$ band. The main advantage of this study lies in the proper accounting for the correlations between flux and anisotropy variations, a restriction to cosmic-ray propagation parameters that were deemed viable in dedicated studies, and an accurate folding with the acceptance distribution of a recent observational study.

We have specifically performed a comparison with recent measurements from the IceCube experiments (Abbasi et al. 2012), which leads us to the following conclusions.

1. Consistent with earlier findings, a cosmic-ray source distribution in the Galaxy that fits the deduced populations of pulsars or SNR, combined with propagation parameters that reproduce the observed secondary-to-primary ratios, will lead to a dipole anisotropy that is higher than observed, even if source discreteness is taken into account.

2. Also in agreement with earlier studies, a shallow energy dependence of diffusion is necessary, thus requiring a reacceleration model to fit the $\mathrm{GeV}$-band boron-to-carbon ratio.

3. For a shallow source distribution, fluctuations arising from source discreteness provide a probability of the order of $10 \%$ that the cosmic-ray anisotropy limits in the highenergy and low-energy bands of the recent IceCube analysis

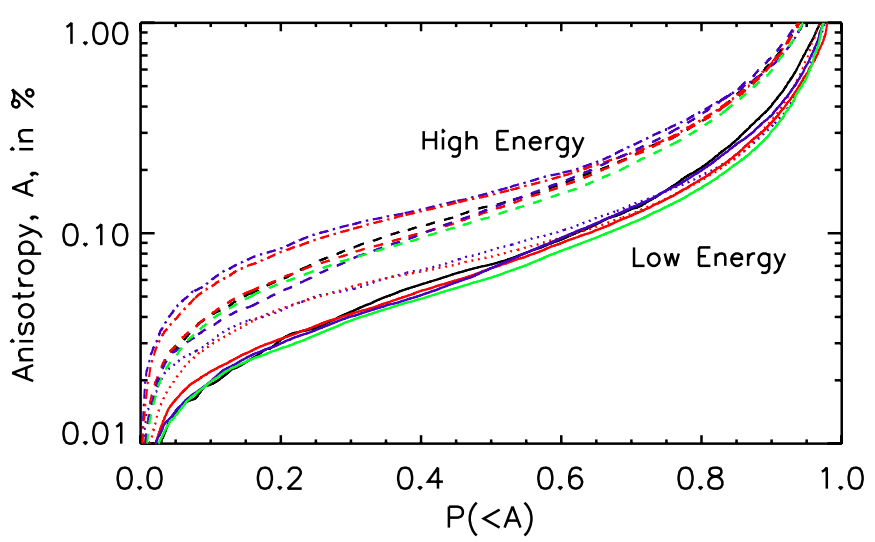

Figure 8. Integral probability, $P(<A)$, to measure an anisotropy lower than a certain threshold, $A$. Solid and dotted lines refer to the low-energy data selection of IceCube, dashed and dot-dashed line are for the high-energy data. The parameters of each curve are listed in Table 5.

(A color version of this figure is available in the online journal.)

can be simultaneously met, approximately independent of the exact choice of source rate, but this is less likely for a large halo size.

4. The locations of the intensity maximum far from the Galactic Center and at different locations for the two energy bands are naturally reproduced when source discreteness is taken into account.

It is unclear what type of cosmic-ray source in the Galaxy would have a distribution in the Galaxy as shallow as required in our study. The question arises whether or not modifications to the description of diffusive transport can render a steep source distribution viable. The critical constraint appears to be imposed by very low dipole anisotropy in the high-energy band of IceCube data, i.e., at approximately $100 \mathrm{TeV}$ energy per nucleon. Is it possible that the extrapolation to this energy of the cosmic-ray propagation parameters does not hold?

Recent data suggest that cosmic-ray spectra (Ahn et al. 2010; Adriani et al. 2011), and the boron-to-carbon ratio (Obermeier et al. 2012), flatten above a few hundred $\mathrm{GeV} \mathrm{nuc}^{-1}$. At this time, we do not have a prevalent interpretation of both the cosmic-ray spectral hardening and the flat $\mathrm{B} / \mathrm{C}$ ratio above a few hundred $\mathrm{GeV}$ nuc $^{-1}$. The spectral hardening of cosmicray primaries could simply be a fluctuation arising from source discreteness, but in that case the boron-to-carbon ratio should fall off more steeply, because the flux of cosmic-ray secondaries fluctuates very little (Büsching et al. 2005). Spectral hardening can also reflect variations in the production spectra of different sources (Brecher \& Burbidge 1972; Büsching et al. 2001; Yuan et al. 2011), in which case the boron-to-carbon ratio would be unaffected. If the upturn in the boron-to-carbon ratio is, on the other hand, due to the fragmentation of primaries and the reacceleration of secondaries inside cosmic-ray sources (Mertsch \& Sarkar 2009; Tomassetti \& Donato 2012), then the energy dependence of the diffusion coefficient cannot be argued to be very shallow. The only possibility that would imply a very low anisotropy is a change of the energy dependence of the diffusion coefficient at a few hundred $\mathrm{GeV}_{\text {nuc }}{ }^{-1}$, e.g., from $\delta=0.25$ to $\delta=0.15$ (Vladimirov et al. 2012; Tomassetti \& Donato 2012). Speculative though that may be, the extrapolation using $\delta=0.15-10^{17} \mathrm{eV}$ may render viable the notion that a significant fraction of the sub-ankle cosmic-ray flux is galactic in origin (e.g., Pohl \& Eichler 2011). 
Recently, Evoli et al. (2012) suggested a common solution to the anisotropy and cosmic-ray gradient problems that involves a strong toroidal magnetic field in the Galaxy. Diffusion perpendicular to the azimuthal magnetic field (with coefficient $D_{\perp} \propto E^{0.6}$, as opposed to $D_{\|} \propto E^{0.3}$ ) would be the dominant transport mechanism, assuming that field-line wandering and large-scale velocity turbulence can be ignored. The authors further posit that $D_{\perp}\left(r_{\mathrm{GC}}\right) \propto 1 / D_{\|}\left(r_{\mathrm{GC}}\right)$ and $D_{\perp} \propto Q\left(r_{\mathrm{GC}}\right)^{\tau}$, where $Q\left(r_{\mathrm{GC}}\right)$ is the density of cosmic-ray sources and $\tau \simeq 0.85$. At least up to $1 \mathrm{TeV}$ particle energy, the predicted radial anisotropy appears to be commensurate with measured values, but it is unclear whether that is also true at the multi-TeV energies considered here.

We expect that the scenario would do little, if anything, to resolve the three-dimensional anisotropy problem for discrete sources (the authors only discuss anisotropy for continuous sources in a two-dimensional scenario). In the direction of fast diffusion, i.e., along the large-scale toroidal magnetic field, we see particles of the same age from sources at larger distance, thus increasing their contribution to the anisotropy. The volume sampled, and thus the number of sources contributing to the local cosmic-ray flux, will also increase, thus reducing the likelihood of a fluctuation toward low anisotropy, but not efficiently enough to overcome anisotropy arising from source discreteness. In the scenario of Evoli et al. (2012), we therefore expect significantly more anisotropy in direction of the ordered magnetic field than in the radial direction. In any case, a careful study is needed to quantify these arguments.

We thank Rasha Abbasi and the IceCube collaboration for providing the proton-only acceptance distribution for their anisotropy analysis. M.P. acknowledges support by the Helmholtz Alliance for Astroparticle Physics, HAP, funded by the Initiative and Networking Fund of the Helmholtz Association. D.E. acknowledges support from the Israel-US Binational Science Foundation, the Israeli Science Foundation, and the Joan and Robert Arnow Chair of Theoretical Astrophysics.

\section{APPENDIX}

\section{ANALYTICAL APPROXIMATION OF ESCAPE}

Mirror sources modify the $z$-dependent term as

$$
\begin{aligned}
& \exp \left[-\frac{\left(z-z_{s}\right)^{2}}{4 D \tau}\right] \longrightarrow G=\sum_{n=-\infty}^{\infty}(-1)^{n} \\
& \quad \times \exp \left[-\frac{\left[z-2 n H-(-1)^{n} z_{s}\right]^{2}}{4 D \tau}\right]
\end{aligned}
$$

that can be rewritten as

$$
\begin{aligned}
G & =\exp \left[-\frac{\left(z-z_{s}\right)^{2}}{4 D \tau}\right] \times\left(1+\sum_{n \neq 0}(-1)^{n}\right. \\
& \left.\times \exp \left[\frac{2 n H\left[z-(-1)^{n} z_{s}-n H\right]-z z_{s}\left(1-(-1)^{n}\right)}{2 D \tau}\right]\right) .
\end{aligned}
$$

The solution is inconvenient because one needs to sum over many terms to find the convergence for all $t$. We are interested in the intermittency behavior and thus need to account for many millions of cosmic-ray sources in the Galaxy, which makes computational efficiency imperative. We can expand the exponential in the series to give

$$
\begin{aligned}
& f=\exp \left[\frac{4 n H\left[z-(-1)^{n} z_{s}\right]-4 n^{2} H^{2}-2 z z_{s}\left(1-(-1)^{n}\right)}{4 D \tau}\right] \\
& \simeq \exp \left[-\frac{n H\left[(-1)^{n} z_{s}+n H\right]}{D \tau}\right] \times\left(1+z A+\frac{z^{2} A^{2}}{2}+\cdots\right),
\end{aligned}
$$

where

$$
A=\frac{2 n H-z_{s}\left(1-(-1)^{n}\right)}{2 D \tau} .
$$

The last factor in brackets is essentially unity, unless $H^{2} \gg D \tau$, in which case the exponential yields zero because its argument is always at least a factor $H / z$ larger as the $z$-dependent terms in the last factor.

We may now expand the remaining exponential for small $z_{s}$ to find

$$
\begin{aligned}
f \simeq & \exp \left[-\frac{n^{2} H^{2}}{D \tau}\right] \\
& \times\left(1-(-1)^{n} z_{s} \frac{n H}{D \tau}+\frac{1}{2}\left[\frac{z_{s} n H}{D \tau}\right]^{2}+\cdots\right),
\end{aligned}
$$

where summation over $\pm n$ removes the first-order term and otherwise yields a factor two. Hence,

$$
\begin{aligned}
G \simeq & \exp \left[-\frac{\left(z-z_{s}\right)^{2}}{4 D \tau}\right] \times\left[1+2 \sum_{n=1}^{\infty}(-1)^{n} \exp \left[-\frac{n^{2} H^{2}}{D \tau}\right]\right. \\
& \left.\times\left(1+\frac{1}{2}\left[\frac{z_{s} n H}{D \tau}\right]^{2}\right)\right] .
\end{aligned}
$$

As for $z$, the second-order term in $z_{s}$ can become relevant only when the exponential is practically zero and the term in brackets unity. Therefore, it can be neglected. A nice analytical approximation for the term in brackets is given by

$$
\begin{aligned}
G & \simeq \exp \left[-\frac{\left(z-z_{s}\right)^{2}}{4 D \tau}\right](1+1.5 x)^{1.25} \exp \left[-(1.5 x)^{0.97}\right] \\
x & =\frac{2 D \tau}{H^{2}}
\end{aligned}
$$

which deviates from the true solution by less than $7.2 \%$ for $x \leqslant 8$, for which the term in brackets is down to $3.6 \times 10^{-4}$.

\section{REFERENCES}

Abbasi, R., Abdou, Y., Abu-Zayyad, T., et al. 2010, ApJL, 718, L194 Abbasi, R., Abdou, Y., Abu-Zayyad, T., et al. 2012, ApJ, 746, 33 Abdo, A. A., Allen, B. T., Aune, T., et al. 2009, ApJ, 698, 2121 Ackermann, M., Ajello, M., Atwood, W. B., et al. 2010, PhRvD, 82, 092003 Ackermann, M., Ajello, M., Atwood, W. B., et al. 2012, ApJ, 750, 3 Ackermann, M., Ajello, M., Baldini, L., et al. 2011, ApJ, 726, 81 Adriani, O., Barbarino, G. C., Bazilevskaya, G. A., et al. 2011, Sci, 332, 69 Aglietta, M., Alekseenko, V. V., Alessandro, B., et al. 2009, ApJL, 692, L130 Ahn, H. S., Allison, P., Bagliesi, M. G., et al. 2010, ApJL, 714, L89 Amenomori, M., Ayabe, S., Bi, X. J., et al. 2006, Sci, 314, 439 Blasi, P., \& Amato, E. 2012a, JCAP, 01, 010

Blasi, P., \& Amato, E. 2012b, JCAP, 01, 011

Brecher, K., \& Burbidge, G. R. 1972, ApJ, 174, 253 
Büsching, I., Kopp, A., Pohl, M., et al. 2005, ApJ, 619, 314

Büsching, I., Pohl, M., \& Schlickeiser, R. 2001, A\&A, 377, 1056

Case, G. L., \& Bhattacharya, D. 1998, ApJ, 504, 761

Di Sciascio, G., \& for the ARGO-YBJ Collaboration 2012, arXiv:1202.3379

Evoli, C., Gaggero, D., Grasso, D., \& Maccione, L. 2012, PhRvL, 108, 211102

Giacinti, G., Kachelrieß, M., \& Semikoz, D. V. 2012, PhRvL, 108, 261101

Giacinti, G., \& Sigl, G. 2012, PhRvL, 109, 071101

Ginzburg, V. L., \& Syrovatskii, S. I. 1964, The Origin of Cosmic Rays (New York: Macmillan)

Green, D. A. 2012, in AIP Conf. Series 1505, High Energy Gamma-ray Astronomy: 5th International Meeting on High Energy Gamma-ray Astronomy, ed. F. A. Aharonian, W. Hoffman, \& F. M. Rieger (Heidelberg, Germany: AIP), 5

Guillian, G., Hosaka, J., Ishihara, K., et al. 2007, PhRvD, 75, 062003

Hörandel, J. R. 2003, APh, 19, 193

Kardashev, N. S. 1962, SvA, 6, 317

Lorimer, D. R. 2004, in IAU Symp. 218, Young Neutron Stars and Their Environments, ed. F. Camilo \& B. M. Gaensler (San Francisco, CA: ASPL), 105
Mertsch, P. 2011, JCAP, 02, 031

Mertsch, P., \& Sarkar, S. 2009, PhRvL, 103, 081104

Obermeier, A., Boyle, P., Hörandel, J., \& Müller, D. 2012, ApJ, 752, 69

Pohl, M., \& Eichler, D. 2011, ApJ, 742, 114

Pohl, M., \& Esposito, J. A. 1998, ApJ, 507, 327

Pohl, M., Perrot, C., Grenier, I., \& Digel, S. 2003, A\&A, 409, 581

Ptuskin, V. S., Jones, F. C., Seo, E. S., \& Sina, R. 2006, AdSpR, 37, 1909

Putze, A., Derome, L., \& Maurin, D. 2010, A\&A, 516, A66

Strong, A. W., Moskalenko, I. V., \& Reimer, O. 2000, ApJ, 537, 763

Strong, A. W., Moskalenko, I. V., Reimer, O., Digel, S., \& Diehl, R. 2004, A\&A, 422, L47

Tomassetti, N., \& Donato, F. 2012, A\&A, 544, A16

Trotta, R., Jóhannesson, G., Moskalenko, I. V., et al. 2011, ApJ, 729, 106

Vladimirov, A. E., Jóhannesson, G., Moskalenko, I. V., \& Porter, T. A. 2012, ApJ, 752, 68

Yoon, Y. S., Ahn, H. S., Allison, P. S., et al. 2011, ApJ, 728, 122

Yuan, Q., Zhang, B., \& Bi, X.-J. 2011, PhRvD, 84, 043002

Zhang, J., Zhang, Y., Cui, S., \& Argo-YbJ Collaboration 2010, 38th COSPAR Scientific Assembly, 38, 2707 